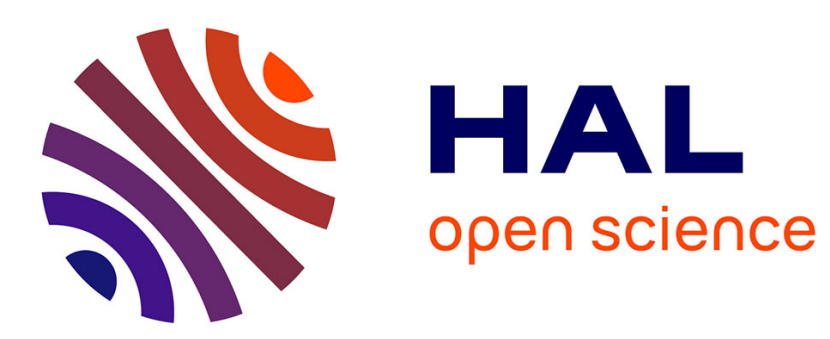

\title{
FEM-based Static Posture Planning for a Humanoid Robot on Deformable Contact Support
}

\author{
Karim Bouyarmane, Abderrahmane Kheddar
}

\section{To cite this version:}

Karim Bouyarmane, Abderrahmane Kheddar. FEM-based Static Posture Planning for a Humanoid Robot on Deformable Contact Support. Humanoids, Oct 2011, Bled, Slovenia. pp.487-492, 10.1109/Humanoids.2011.6100848 . lirmm-00765818

\section{HAL Id: lirmm-00765818 https://hal-lirmm.ccsd.cnrs.fr/lirmm-00765818}

Submitted on 16 Dec 2012

HAL is a multi-disciplinary open access archive for the deposit and dissemination of scientific research documents, whether they are published or not. The documents may come from teaching and research institutions in France or abroad, or from public or private research centers.
L'archive ouverte pluridisciplinaire HAL, est destinée au dépôt et à la diffusion de documents scientifiques de niveau recherche, publiés ou non, émanant des établissements d'enseignement et de recherche français ou étrangers, des laboratoires publics ou privés. 


\title{
FEM-based Static Posture Planning for a Humanoid Robot on Deformable Contact Support
}

\author{
Karim Bouyarmane and Abderrahmane Kheddar
}

\begin{abstract}
In this paper we extend our previous work on solving the inverse kinematics problem for a humanoid robot in general multi-contact stances under physical limitations and static equilibrium constraints, to the case in which the contact is made on a non-rigid deformable environment support. We take a finite element approach to solve the static equilibrium equations for the system made of the robot and the deformable support within the linear elasticity model. Example simulation results show the humanoid robot HRP-2 taking contact support with hand or foot link on a deformable cube.
\end{abstract}

\section{INTRODUCTION}

In [1] we presented an optimization-based solution for the inverse kinematics problem on non-horizontal non-coplanar frictional multi-contact stances for a humanoid robot subject to joint and torque limits under the static equilibrium constraint. This work was subsequently used within a contactbefore-motion planning framework [2] that extended the seminal works of [3], [4] to general multi-agent systems for solving indifferently locomotion and manipulation planning problems centred around the humanoid robot.

One common assumption in all of these works is the rigidity hypothesis, for both the robot links and the environment objects. Our aim in this work is to further extend the capabilities of these contact-before-motion planners to cope with deformable objects in the environment under the linear elasticity hypothesis. This can be made possible if the underlying inverse kinematics solver under static equilibrium constraint can deal with such linear elasticity models. Thus we focus on this latter task, extending the solver presented in [1] to the case in which the contact prints are positioned on a surface belonging to a deformable object in the environment.

The approach we choose to solve for the static equilibrium equations of the elastic material is based on the finite element method. The deformation of the contact support is related to the corresponding position of the supported link of the robot and is as such a function of the configuration of the robot. This deformation generates reaction forces that have to be taken into account in the equilibrium equation of the robot. The main contribution of this work is thus to relate the induced deformation forces to the configuration of the robot in a way that will allow us to derive the gradient of the extended static equilibrium constraint fed to the non-linear constrained optimization solver.

The authors are with CNRS-AIST JRL (Joint Robotics Laboratory) UMI3218/CRT, AIST, Tsukuba, Japan; and with CNRS-University of Montpellier 2 LIRMM, Montpellier, France. \{karim.bouyarmane, abderrahmane.kheddar\}@aist.go.jp
The rest of the paper is organized as follows. In Section II we introduce the notations used by recalling the finite element method for linear elasticity models. We then write the constraint and its gradient in Section III which constitutes the main development of the paper. Example applications are presented in Section IV, before concluding the paper by discussing limitations and perspectives in Section V.

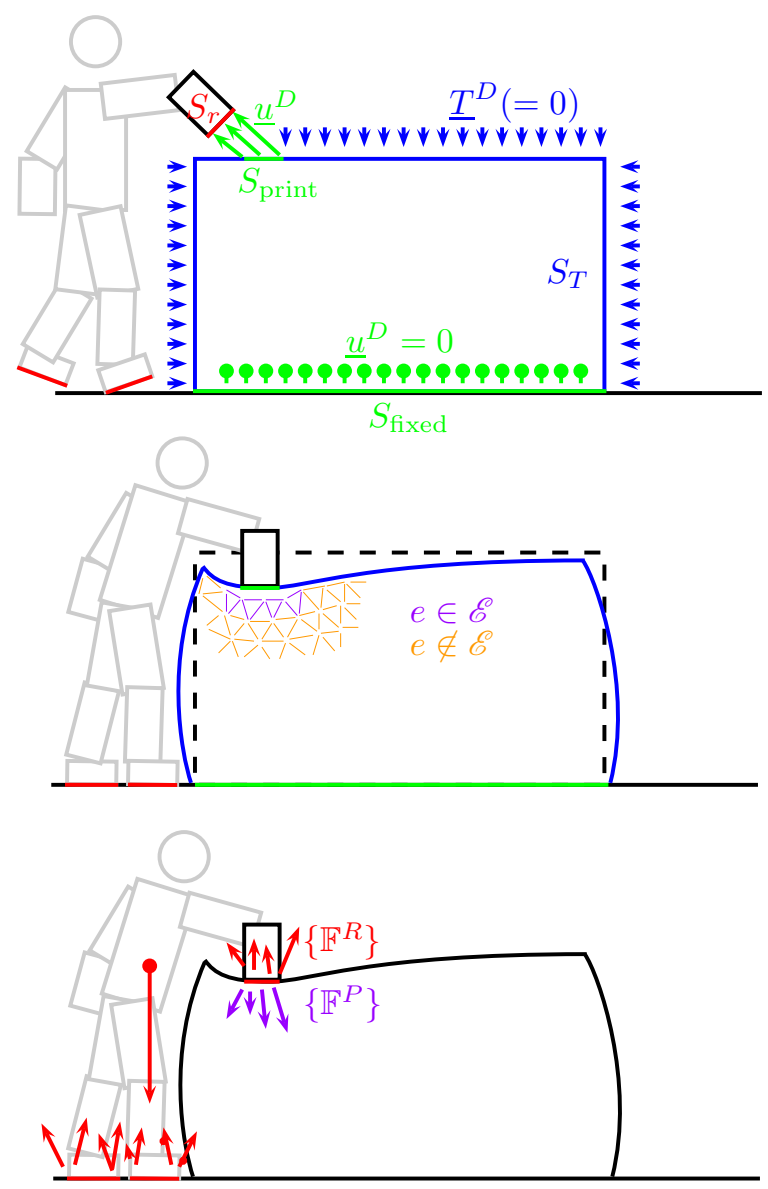

Fig. 1. Overview illustration of the method.

\section{The Finite Element Method}

We first recall the finite element method we use to formulate and solve the problem. This section is mainly adapted from the reference textbook [5] that we reproduce here in order to introduce the notations that we need for the sake of our formulation. ${ }^{1}$

\footnotetext{
${ }^{1}$ We encourage the reader familiar with the method to still go through this section as a minimum requirement to understand the notations and reasoning of the rest of the paper.
} 
So let us consider a solid object that occupies in the Euclidean space a volume denoted $\Omega$ subject to the behaviour model of linear elasticity under the small deformation hypothesis. The following equations govern the static equilibrium of the object:

$$
\begin{gathered}
\underline{\underline{\varepsilon}}(\underline{x})=\frac{1}{2}\left(\nabla \underline{u}+\nabla^{T} \underline{u}\right)(\underline{x}), \\
\operatorname{div} \underline{\underline{\sigma}}(\underline{x})+\rho \underline{f}(\underline{x})=0, \\
\underline{\underline{\sigma}}(\underline{x})=\underline{\underline{\underline{\underline{A}}}}: \underline{\underline{\varepsilon}}(\underline{x}),
\end{gathered}
$$

where $\underline{x} \in \Omega, \underline{\underline{\varepsilon}}$ is the strain tensor field, $\underline{u}$ is the displacement field, $\underline{\underline{\sigma}}$ is the Cauchy stress tensor field, $\underline{\underline{\mathcal{A}}}$ is the elasticity tensor, $\rho$ is the mass density of the material, $f$ is the body force density field. The boundary conditions for a well-posed problem are specified as:

$$
\begin{aligned}
\underline{\sigma}(\underline{x}) \cdot \underline{n}(\underline{x})=\underline{T}^{D}(\underline{x}) & \left(\underline{x} \in S_{T}\right), \\
\underline{u}(\underline{x})=\underline{u}^{D}(\underline{x}) & \left(\underline{x} \in S_{u}\right),
\end{aligned}
$$

where $\underline{T}^{D}$ and $\underline{u}^{D}$ are respectively the prescribed surface force density (traction) and prescribed displacement fields on the the surfaces $S_{T}$ and $S_{u}$ that constitute a partition of the frontier $\partial \Omega\left(S_{T} \cap S_{u}=\varnothing\right.$ and $\left.S_{T} \cup S_{u}=\partial \Omega\right)$, and $\underline{n}(\underline{x})$ is the unit normal to the surface $\partial \Omega$ at the point $\underline{x}$.

By applying the virtual work principle, or by following a variational approach minimizing potential energy, we can get to the weak formulation of the problem, in which we look for a displacement field $\underline{u}$ satisfying (5) such that:

$$
\int_{\Omega} \underline{\underline{\varepsilon}}[\underline{u}]: \underline{\underline{\underline{\underline{\mathcal{A}}}}}: \underline{\underline{\varepsilon}}[\underline{w}] d V=\int_{\Omega} \rho \underline{f} \cdot \underline{w} d V+\int_{S_{T}} \underline{T}^{D} \cdot \underline{w} d S,
$$

for all the virtual displacement fields $\underline{w}$ that are zero on the surface $S_{u}$, where we have used the notation

$$
\underline{\underline{\varepsilon}}[\underline{v}]=\frac{1}{2}\left(\nabla \underline{v}+\nabla^{T} \underline{v}\right) .
$$

We approximate the domain $\Omega$ by a domain $\Omega_{h}=\cup_{e} E_{e}$ $\left(1 \leq e \leq N_{E}\right)$ made of isoparametric elements $E_{e}$ of characteristic dimension $h$ (the subscript $h$ will be used to make distinction between the exact problem and the approximated problem) that constitute a mesh of $\Omega_{h}$, the nodes of which are denoted $\underline{x}^{(n)}$ (globally within the whole mesh, or $\underline{x}_{e}^{(k)}, 1 \leq k \leq n_{e}$, locally within each element $e$ ). The position of a point $\underline{x} \in E_{e}$ is interpolated from the positions of the nodes of the element using the local shape functions $N_{k}$

$$
\underline{x}=\sum_{k=1}^{n_{e}} N_{k}(\underline{a}) \underline{x}_{e}^{(k)},
$$

where $\underline{a}$ is a parameter varying in a reference non-deformed "unit" element $\Delta_{e}$, and we choose to interpolate accordingly an arbitrary displacement field $\underline{v}_{h}$ on the nodal displacements $\underline{v}^{(k)}$ using the same interpolation

$$
\underline{v}_{h}=\sum_{k=1}^{n_{e}} N_{k}\left(\underline{a} \underline{v}^{(k)} .\right.
$$

We also introduce an injective index function $\operatorname{dof}(n, j)$ such that $\operatorname{dof}(n, j)>0$ if the coordinate $j$ (along the basis vector $\underline{e}_{j}$ ) of the node $\underline{x}^{(n)}$ is free, meaning that the node $\underline{x}^{(n)}$ does not belong to the prescribed-displacement surface $S_{u, h} \subset \partial \Omega_{h}$, and $\operatorname{dof}(n, j) \leq 0$ otherwise.

Finally we apply the Galerkin method. We look for a displacement field of the form

$$
\underline{u}_{h}(\underline{x})=\underline{u}_{h}^{(D)}(\underline{x})+\underline{u}_{h}^{(0)}(\underline{x}),
$$

where the fields $\underline{u}_{h}^{(D)}$ and $\underline{u}_{h}^{(0)}$, respectively satisfying the boundary condition (5) and vanishing on the surface $S_{u, h}$, are interpolated as

$$
\begin{gathered}
\underline{u}_{h}^{(D)}(\underline{x})=\sum_{(n, j) \mid \operatorname{dof}(n, j) \leq 0} \tilde{N}_{n}(\underline{x}) u_{j}^{(D)}\left(\underline{x}^{(n)}\right) \underline{e}_{j}, \\
\underline{u}_{h}^{(0)}(\underline{x})=\sum_{(n, j) \mid \operatorname{dof}(n, j)>0} \tilde{N}_{n}(\underline{x}) u_{j}^{(n)} \underline{e}_{j},
\end{gathered}
$$

where $\tilde{N}_{n}$ are the global shape functions constructed from the functions $N_{k}$ in (8) so as to represent the position of a point expressed in the whole domain $\Omega_{h}$. The virtual displacement field defined in the weak formulation (6) takes the form

$$
\underline{w}(\underline{x})=\sum_{(n, j) \mid \operatorname{dof}(n, j)>0} \tilde{N}_{n}(\underline{x}) w_{j}^{(n)} \underline{e}_{j},
$$

and the weak formulation (6) amounts now to finding a field $\underline{u}_{h}^{(0)}$ of the form (12) such that for every field $\underline{w}$ of the form (13) we have

$$
\begin{aligned}
\int_{\Omega_{h}} \underline{\underline{\varepsilon}}\left[\underline{u}_{h}^{(0)}\right]: \underline{\underline{\underline{\underline{\mathcal{A}}}}}: & \underline{\underline{\varepsilon}}[\underline{w}] d V=-\int_{\Omega_{h}} \underline{\underline{\varepsilon}}\left[\underline{u}_{h}^{(D)}\right]: \underline{\underline{\underline{\underline{\mathcal{A}}}}: \underline{\underline{\varepsilon}}[\underline{w}] d V} \\
& +\int_{\Omega_{h}} \rho \underline{f} \cdot \underline{w} d V+\int_{S_{T, h}} \underline{T}^{D} \cdot \underline{w} d S .
\end{aligned}
$$

By gathering the free nodes displacements coordinates $u_{j}^{(n)}$ and $w_{j}^{(n)}(\operatorname{dof}(n, j)>0)$ respectively in the vectors $\left\{\mathbb{U}^{F}\right\}$ and $\{\mathbb{W}\}$, the formulation (14) takes the following linear system form

$$
\{\mathbb{W}\}^{T}\left[\mathbb{K}^{F}\right]\left\{\mathbb{U}^{F}\right\}=\{\mathbb{W}\}^{T}\{\mathbb{F}\},
$$

or equivalently

$$
\left[\mathbb{K}^{F}\right]\left\{\mathbb{U}^{F}\right\}=\{\mathbb{F}\},
$$

where the rigidity matrix $\left[\mathbb{K}^{F}\right]$ and generalized nodal forces $\{\mathbb{F}\}$ are defined as sums of elementary integrals over the elements $E_{e}$ through identification respectively in

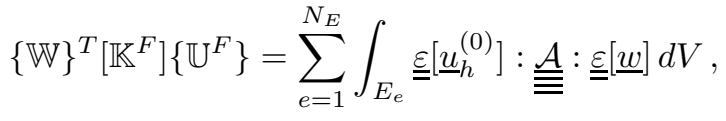

and

$$
\begin{aligned}
\{\mathbb{W}\}^{T}\{\mathbb{F}\}= & \left.\sum_{e=1}^{N_{E}}-\int_{E_{e}} \underline{\underline{\varepsilon}} \underline{\underline{[}} \underline{\underline{u}}_{h}^{(D)}\right]: \underline{\underline{\underline{\underline{A}}}: \underline{\underline{\varepsilon}}} \underline{\underline{w}]} d V \\
& +\int_{E_{e}} \rho \underline{f} \cdot \underline{\underline{w}} d V+\int_{S_{T, h} \cap E_{e}} \underline{T}^{D} \cdot \underline{w} d S .
\end{aligned}
$$




\section{The Voigt Notation}

Let us differentiate the two relations (8) and (9)

$$
\begin{aligned}
d \underline{x} & =\underline{\underline{J}}(\underline{a}) d \underline{a}, \\
d \underline{v}_{h} & =\underline{\underline{H}}(\underline{a}) d \underline{a} .
\end{aligned}
$$

This allows us to rewrite the relation (7) applied to the field $\underline{v}_{h}$ as

$$
\underline{\underline{\varepsilon}}\left[\underline{v}_{h}\right](\underline{x})=\frac{1}{2}\left(\underline{\underline{H}}(\underline{a}) \cdot \underline{\underline{J}}^{-1}(\underline{a})+\left(\underline{\underline{H}}(\underline{a}) \cdot \underline{\underline{J}}^{-1}(\underline{a})\right)^{T}\right) .
$$

By introducing the Voigt representations of the symmetric tensors $\underline{\underline{\varepsilon}}$ and $\underline{\underline{\sigma}}$, which are the $\mathbb{R}^{6}$ vectors containing the 6 independents components of the two tensors

$$
\begin{aligned}
& \{\sigma\}=\left\{\begin{array}{llllll}
\sigma_{11} & \sigma_{22} & \sigma_{33} & \sigma_{12} & \sigma_{13} & \sigma_{23}
\end{array}\right\}^{T},
\end{aligned}
$$

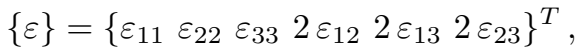

the relation (21) takes the form

$$
\{\varepsilon\}=\left[B_{e}(\underline{a})\right]\left\{\mathbb{V}_{e}\right\},
$$

where $\left\{\mathbb{V}_{e}\right\}$ is the $\mathbb{R}^{3 n_{e}}$ vector concatenating the nodal displacements $\underline{v}^{(k)}$ and $\left[B_{e}(\underline{a})\right]$ is a $6 \times 3 n_{e}$ matrix obtained through identification in (21). Moreover, the relation (3) simplifies into

$$
\{\sigma\}=[A]\{\varepsilon\},
$$

where $[A]$ is the $6 \times 6$ matrix written in terms of the Lamé parameters $\lambda$ and $\mu$ for an isotropic homogeneous material

$$
[A]=\left[\begin{array}{cccccc}
\lambda+2 \mu & \lambda & \lambda & 0 & 0 & 0 \\
\lambda & \lambda+2 \mu & \lambda & 0 & 0 & 0 \\
\lambda & \lambda & \lambda+2 \mu & 0 & 0 & 0 \\
0 & 0 & 0 & \mu & 0 & 0 \\
0 & 0 & 0 & 0 & \mu & 0 \\
0 & 0 & 0 & 0 & 0 & \mu
\end{array}\right]
$$

These relations allows for a simple evaluation of the elementary integrals in (17) and (18) using a Gauss-pointbased numerical method.

\section{Formulation of the Planning Problem}

Let us now consider the problem of a humanoid robot in multi-contact stance with its environment, in which one of the contacts (we will refer to it as the "deformable contact") is made on the surface of the deformable object introduced in the previous section. See Fig. 1. Let the corresponding contact surface on the robot be denoted $S_{r}$, which is a planar surface defined on a link $l$ of the robot $r$. The desired relative position and orientation of the deformable contact $(x, y, \theta) \in S E(2)$ define a contact print, that is the image of the surface $S_{r}$ projected onto $\partial \Omega$ and positioned according to $(x, y, \theta)$. Let this contact print be denoted $S_{\text {print }} \subset \partial \Omega$, and the corresponding bijective projection mapping $p_{\text {print }}: S_{r} \rightarrow$ $S_{\text {print }}$, which is simply a rigid transformation. Furthermore, a portion $S_{\text {fixed }}$ of the frontier $\partial \Omega$ is fixed on the environment, for instance the base of the deformable object contacting the

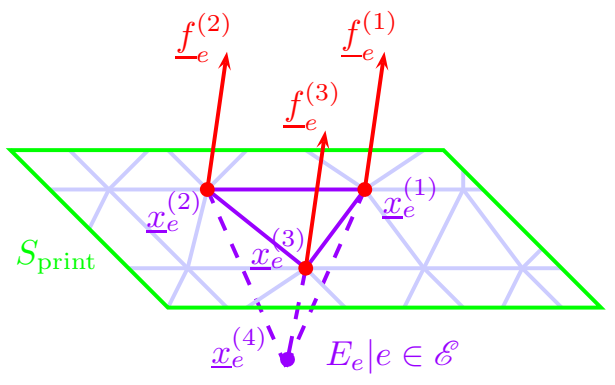

Fig. 2. Nodal reaction forces.

rigid floor. Let $\mathscr{P}$ and $\mathscr{F}$ be the subsets of the surface nodes of the mesh $\underline{x}^{(n)}$ that lie inside $S_{\text {print }}$ and $S_{\text {fixed }}$ respectively

$$
\begin{aligned}
& \mathscr{P}=\left\{\underline{x}^{(n)} \mid \underline{x}^{(n)} \in S_{\text {print }}\right\}, \\
& \mathscr{F}=\left\{\underline{x}^{(n)} \mid \underline{x}^{(n)} \in S_{\text {fixed }}\right\} .
\end{aligned}
$$

The prescribed-displacement surface in the boundary condition (5) is in this case $S_{u}=S_{\text {print }} \cup S_{\text {fixed }}$, and the prescribed nodal displacements are

$$
\underline{u}^{(D)}\left(\underline{x}^{(n)}\right)= \begin{cases}0 & \text { if } \underline{x}^{(n)} \in \mathscr{F}, \\ p_{\text {print }}^{-1}\left(\underline{x}^{(n)}\right)-\underline{x}^{(n)} & \text { if } \underline{x}^{(n)} \in \mathscr{P} .\end{cases}
$$

On the remaining surface $S_{T}=\partial \Omega \backslash\left(S_{\text {print }} \cup S_{\text {fixed }}\right)$ the prescribed traction is set to zero

$$
\underline{T}^{D}(\underline{x})=0 \quad\left(\underline{x} \in S_{T}\right)
$$

and the body force density is also set to zero

$$
\underline{f}(\underline{x})=0 \quad(\underline{x} \in \Omega) .
$$

In these conditions, by concatenating the prescribed nodal displacements $\underline{u}^{(D)}\left(\underline{x}^{(n)}\right)$ into the vector $\left\{\mathbb{U}^{D}\right\}$, the nodal forces vector (18) takes the form

$$
\{\mathbb{F}\}=-\left[\mathbb{K}^{D}\right]\left\{\mathbb{U}^{D}\right\},
$$

where the matrix $\left[\mathbb{K}^{D}\right]$ is defined through identification in

$$
\{\mathbb{W}\}^{T}\left[\mathbb{K}^{D}\right]\left\{\mathbb{U}^{D}\right\}=\sum_{e=1}^{N_{E}} \int_{E_{e}} \underline{\underline{\varepsilon}}\left[\underline{u}_{h}^{(D)}\right]: \underline{\underline{\underline{\underline{A}}}}: \underline{\underline{\varepsilon}}[\underline{w}] d V .
$$

Finally equation (16) reduces to

$$
\left[\mathbb{K}^{F}\right]\left\{\mathbb{U}^{F}\right\}+\left[\mathbb{K}^{D}\right]\left\{\mathbb{U}^{D}\right\}=0,
$$

which can be rewritten as

$$
\{\mathbb{U}\}=\left\{\begin{array}{l}
\mathbb{U}^{F} \\
\mathbb{U}^{D}
\end{array}\right\}=\left[\begin{array}{c}
-\left[\mathbb{K}^{F}\right]^{-1}\left[\mathbb{K}^{D}\right] \\
I
\end{array}\right]\left\{\mathbb{U}^{D}\right\},
$$

the vector $\{\mathbb{U}\}$ containing now the displacements of all the nodes of the mesh, and $I$ being the identity matrix of dimension $\operatorname{dim}\left(\left\{\mathbb{U}^{D}\right\}\right)$. We rewrite this latter equation in a more compact form

$$
\{\mathbb{U}\}=[\mathbb{K}]\left\{\mathbb{U}^{D}\right\} .
$$




\section{Nodal Reaction Forces}

We would like now compute the nodal reaction forces $\left\{\mathbb{F}^{R}\right\}$ that are applied through the nodes of the contact print $\mathscr{P}$ on the contact link $l$ of the robot $r$. See Fig. 2. First let us define what we mean by such nodal reaction forces. Let $\underline{T}^{P}$ be the traction that is applied on the the deformable object through the surface $S_{\text {print }}$. For a point $\underline{x} \in S_{\text {print }}$ we have

$$
\underline{T}^{P}(\underline{x})=\underline{\sigma}(\underline{x}) \cdot \underline{n}(\underline{x}) \text {. }
$$

We approximate the the surface print $S_{\text {print }}$ by $S_{\text {print } h}$ defined as the union of the surfaces of the elements that have all of their frontier nodes belonging to $\mathscr{P}$. These elements are members of the set

$$
\mathscr{E}=\left\{e \in\left\{1, \ldots, N_{E}\right\} \mid E_{e} \cap \partial \Omega_{h} \subset S_{\text {print }}\right\},
$$

and thus $S_{\text {print }, h}$ is

$$
S_{\text {print }, h}=\bigcup_{e \in \mathscr{E}} E_{e} \cap \partial \Omega_{h} .
$$

For every $e \in \mathscr{E}$, we would like to compute the traction $\underline{T}^{P}(\underline{x})$ when $\underline{x}$ varies in $E_{e} \cap \partial \Omega_{h}$. Since we chose to use tetrahedron elements, the matrix $\left[B_{e}(\underline{a})\right]$ defined in (24) can be shown to be independent of the parameter $\underline{a},\left[B_{e}(\underline{a})\right]=$ $\left[B_{e}\right]$, and the stress field $\underline{\underline{\sigma}}(\underline{x})$ is thus constant within every element $E_{e}, \underline{\underline{\sigma}}(\underline{x})=\underline{\underline{\sigma}}_{e}$. Since $E_{e} \cap \partial \Omega_{h}$ reduces in this case to a planar triangle, the normal $\underline{n}(\underline{x})$ is constant throughout $E_{e} \cap \partial \Omega_{h}$, we denote it $\underline{n}_{e}$, and subsequently $\underline{T}^{P}(\underline{x})$ is also constant throughout $E_{e} \cap \partial \Omega_{h}$, we denote it $\underline{T}_{e}^{P}$. The nodal surface forces $\left\{\mathbb{F}_{e}^{P}\right\}$ are defined such that for every virtual displacement field $\underline{w}(\underline{x}), x \in E_{e} \cap \partial \Omega_{h}$ interpolating the nodal displacements $\left\{\mathbb{W}_{e}^{P}\right\}$ of the three surface triangle nodes through the interpolation (9) we have

$$
\left\{\mathbb{W}_{e}^{P}\right\}^{T}\left\{\mathbb{F}_{e}^{P}\right\}=\int_{E_{e} \cap \partial \Omega_{h}} \underline{T}_{e}^{P} \cdot \underline{w} d S .
$$

If $\alpha_{e}$ denotes the area of the triangle $E_{e} \cap \partial \Omega_{h}$, we can show that identification in this latter relation leads to

$$
\left\{\mathbb{F}_{e}^{P}\right\}=\frac{\alpha_{e}}{3} N \underline{\underline{\sigma}}_{e} \cdot \underline{n}_{e},
$$

where $N$ is a duplication matrix

$$
N=\left[\begin{array}{lll}
I_{3 \times 3} & I_{3 \times 3} & I_{3 \times 3}
\end{array}\right]^{T} .
$$

$\left\{\mathbb{F}_{e}^{R}\right\}$, the contribution of the element $e \in \mathscr{E}$ to the nodal reaction forces $\left\{\mathbb{F}^{R}\right\}$, is the opposite of this vector

$$
\left\{\mathbb{F}_{e}^{R}\right\}=-\left\{\mathbb{F}_{e}^{P}\right\}=-\frac{\alpha_{e}}{3} N \underline{\underline{\sigma}} \cdot \underline{n}_{e},
$$

The application points of $\left\{\mathbb{F}_{e}^{R}\right\}$ are the vertices $\left(\underline{x}_{e}^{(1)}, \underline{x}_{e}^{(2)}, \underline{x}_{e}^{(3)}\right)$ of the triangle $E_{e} \cap \partial \Omega_{h}$. Finally the reaction surface force distribution over the triangle $E_{e} \cap \partial \Omega_{h}$ is equivalent from a virtual work point of view to the set of three point forces

$$
\left\{\begin{array}{ll}
\underline{f}_{e}^{(1)}=-\frac{\alpha_{e}}{3} \underline{\sigma}_{e} \cdot \underline{n}_{e} & \text { applied at } \underline{x}_{e}^{(1)} \\
\underline{f}_{e}^{(2)}=-\frac{\alpha_{e}}{3} \underline{\sigma}_{e} \cdot \underline{n}_{e} & \text { applied at } \underline{x}_{e}^{(2)} \\
\underline{f}_{e}^{(3)}=-\frac{\alpha_{e}}{3} \underline{\sigma}_{e} \cdot \underline{n}_{e} & \text { applied at } \underline{x}_{e}^{(3)}
\end{array}\right\} .
$$

\section{The Optimization Approach}

We recall now the approach followed in [1] for solving an inverse problem. if $q$ denotes the configuration of the robot (including the free-flying base component in $S E(3)$ ) and $\Lambda$ the set of non-negative coefficients along the linearised friction cone generators at the contact points, the approach consists in solving the non-linear constrained optimization problem of an arbitrary objective function $\mathrm{obj}^{2}$

$$
\begin{aligned}
\underset{(q, \Lambda)}{\min } & \operatorname{obj}(q, \Lambda) \\
\text { under } & \text { joint limits, } \\
& \text { torque limits, } \\
& \text { friction cone, } \\
& \text { and static equilibrium constraints. }
\end{aligned}
$$

Taking into account the deformable contact is straightforward by adding the forces (44) to the set of contact forces applied on the robot in the formulation of the torque limits and static equilibrium constraints (47) and (49) of the formulation (45). One difficulty arises in computing the contribution of these forces to the gradient of these two constraints (47) and (49).

So let us consider one of these forces $\underline{f}_{e}^{(j)}(e \in \mathscr{E}$ and $j \in\{1,2,3\})$ and try to explicit its dependency on the configuration of the robot $q$. We have

$$
\underline{f}_{e}^{(j)}(q)=-\frac{\alpha_{e}}{3} \underline{\sigma}_{e}(q) \cdot \underline{n}_{e}(q) .
$$

Note that since the projection operator $p_{\text {print }}$ is a rigid transformation the area of the element frontier triangle $\alpha_{e}$ is constant and does not depend on $q$. Let $[D]$ be the $9 \times 6$ duplication matrix

$$
[D]=\left[\begin{array}{llllll}
1 & 0 & 0 & 0 & 0 & 0 \\
0 & 0 & 0 & 1 & 0 & 0 \\
0 & 0 & 0 & 0 & 1 & 0 \\
0 & 0 & 0 & 1 & 0 & 0 \\
0 & 1 & 0 & 0 & 0 & 0 \\
0 & 0 & 0 & 0 & 0 & 1 \\
0 & 0 & 0 & 0 & 1 & 0 \\
0 & 0 & 0 & 0 & 0 & 1 \\
0 & 0 & 1 & 0 & 0 & 0
\end{array}\right],
$$

such that we can write the definition of the Voigt notation (22) of the stress tensor $\left\{\sigma_{e}\right\}$ as a vectorization relation

$$
[D]\left\{\sigma_{e}\right\}=\operatorname{vec}\left(\underline{\sigma}_{e}\right) \text {, }
$$

where $\operatorname{vec}(M)$ means the column vector obtained by concatenating all the columns of $M$ into one column vector [6].

\footnotetext{
${ }^{2}$ The objective function is designed in a way to minimize a distance to a reference posture and to optimize the repartition of contact forces or actuation torques. In the present case an additional weighted component aimed at minimizing the deformation can be added by minimizing the norm of the nodal reaction forces that will are derived in the subsequent development of the paper. This is done in particular in the presented results at the end of the paper.
} 
Since $\underline{\sigma}_{e} \cdot \underline{n}_{e}$ is already a column vector then its vectorization is trivial

$$
\operatorname{vec}\left(\underline{\underline{\sigma}}_{e} \cdot \underline{\underline{n}}_{e}\right)=\underline{\underline{\sigma}}_{e} \cdot \underline{n}_{e} .
$$

The algebra of the vectorization operation tells us that the vectorization of a matrix product can be derived using the Kronecker product operation $\otimes$

$$
\operatorname{vec}\left(M_{1} M_{2}\right)=\left(M_{2}^{T} \otimes I_{k \times k}\right) \operatorname{vec}\left(M_{1}\right),
$$

where $k$ is the number of rows of $M_{1}$. So the relation (53) using (52) becomes

$$
\begin{aligned}
\underline{\underline{\sigma}}_{e} \cdot \underline{n}_{e} & =\operatorname{vec}\left(\underline{\underline{\sigma}}_{e} \cdot \underline{n}_{e}\right), \\
& =\left(\underline{n}_{e}^{T} \otimes I_{3 \times 3}\right) \operatorname{vec}\left(\underline{\underline{\sigma}}_{e}\right), \\
& =\left(\underline{n}_{e}^{T} \otimes I_{3 \times 3}\right)[D]\left\{\sigma_{e}\right\} .
\end{aligned}
$$

Moreover, from (24) and (25) we can write

$$
\left\{\sigma_{e}\right\}=[A]\left[B_{e}\right]\left\{\mathbb{U}_{e}\right\},
$$

where $\left\{\mathbb{U}_{e}\right\}$ are the nodal displacements of the four vertices of the element $E_{e}$, which can be obtained from (36) as

$$
\left\{\mathbb{U}_{e}\right\}=\left[\mathbb{K}_{e}\right]\left\{\mathbb{U}^{D}\right\},
$$

$\left[\mathbb{K}_{e}\right]$ being the matrix extracted by keeping only the 12 rows of the matrix $[\mathbb{K}]$ corresponding to the 12 components $\left\{\mathbb{U}_{e}\right\}$ in $\{\mathbb{U}\}$. Finally we can rewrite an explicit expression of (50)

$$
\underline{f}_{e}^{(j)}(q)=-\frac{\alpha_{e}}{3}\left(\underline{n}_{e}(q)^{T} \otimes I_{3 \times 3}\right)[D][A]\left[B_{e}\right]\left[\mathbb{K}_{e}\right]\left\{\mathbb{U}^{D}(q)\right\} .
$$

The gradient of (60) with respect to $q$ can now be derived based on the two computationally available Jacobian matrices of the contact link $l$ of the robot $r$

$$
\begin{gathered}
\frac{\partial \underline{n}_{e}(q)}{\partial q}, \\
\frac{\partial\left\{\mathbb{U}^{D}(q)\right\}}{\partial q} .
\end{gathered}
$$

(Recall that at the solution $S_{r}=S_{\text {print }}$ and thus $\underline{n}_{e}(q)$ and the non-zero components of $\left\{\mathbb{U}^{D}(q)\right\}$ can be considered as rigidly attached to $S_{r}$ ie. rigidly attached to the link $l$ ). This gradient takes the final form

$$
\begin{aligned}
\frac{\partial \underline{f}_{e}^{j}}{\partial q}= & \\
-\frac{\alpha_{e}}{3}[ & \left.\left(\left[\frac{\partial \underline{n}_{e}}{\partial q_{i}}\right]^{T} \otimes I_{3 \times 3}\right)[D][A]\left[B_{e}\right]\left[\mathbb{K}_{e}\right]\left\{\mathbb{U}^{D}\right\}\right]_{i=1}^{\operatorname{dim}(\mathrm{q})} \\
& -\frac{\alpha_{e}}{3}\left(\underline{n}_{e}^{T} \otimes I_{3 \times 3}\right)[D][A]\left[B_{e}\right]\left[\mathbb{K}_{e}\right] \frac{\partial\left\{\mathbb{U}^{D}\right\}}{\partial q}
\end{aligned}
$$

The gradients of the moment of the force $f_{e}^{(j)}(q)$ and the torques resulting from it follow directly using the Jacobians at the application points that can also be considered as being attached to the surface $S_{r}$ and thus to the link $l$ of the robot $r$

$$
\frac{\partial \underline{x}_{e}^{(j)}(q)}{\partial q} \text {. }
$$

Finally the computation of these gradients allows us to use non-linear optimization solvers such as [7], [8] to solve the problem (45) taking into account the nodal reaction forces.

\section{Simulation Results}

We applied the presented method to an example scenario in which the humanoid robot HRP-2 [9] takes support with both feet on the rigid ground and with a modified hand link on a deformable object.

The deformable object is a simple $1 \mathrm{~m} \times 1 \mathrm{~m} \times 1 \mathrm{~m}$ cube with an isoparametric mesh made of 166 nodes and 570 tetrahedron elements. See Fig. 3. Table I gives the physical properties of the material that constitutes the cube.

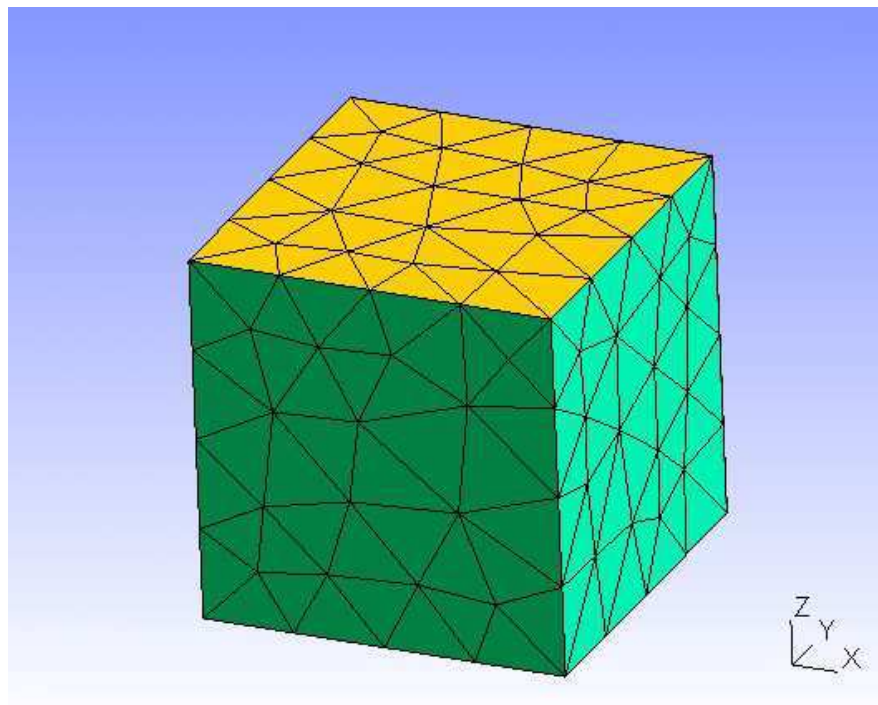

Fig. 3. The mesh of the deformable environment contact support.

\begin{tabular}{|c|c|}
\hline Young's modulus $E$ & $10^{6} \mathrm{~Pa}$ \\
\hline Poisson's ratio $\nu$ & 0.4 \\
\hline Mass density $\rho$ & $10^{3} \mathrm{~kg} / \mathrm{m}^{3}$ \\
\hline
\end{tabular}

TABLE I

PROPERTIES OF THE DEFORMABLE MATERIAL.

Fig. 4 shows the resulting configuration together with snapshots configurations along the optimisation iteration process. Note that we are only interested in the final iterate, the intermediate configurations do not have physical meaning.

In another example scenario, shown in Fig. 5, the HRP-2 robot has its left foot supporting on a rigid object and its right foot supporting on the same deformable cube.

Finally, Fig. 6 shows for the sake of visualization an on-purpose exaggerated deformation resulting from lower Young's modulus of the material constituting the cube. This configuration is not physically valid since the linear elasticity regime should be applied under the small deformation hypothesis, which occurs only in the first case. For large deformations, non-linear approaches such as [10], [11] should be investigated.

As for execution time, the orders of magnitude as reported in [1] range from one to ten seconds per query. Adding the FEM resolution step keeps it in the order of tens of seconds, without any effort devoted to reducing this time in our prototype implementation. 


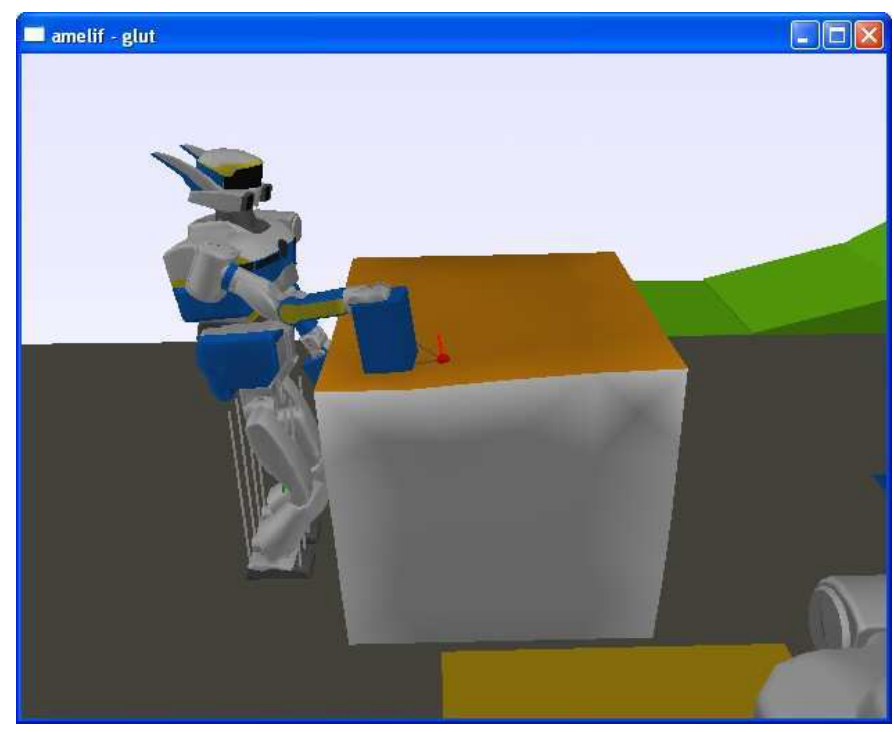

(a) Final result

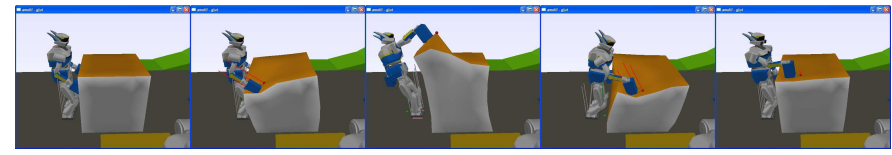

(b) $i=0 / 68 \quad$ (c) $i=1 / 68 \quad$ (d) $i=20 / 68$ (e) $i=50 / 68$ (f) $i=68 / 68$

Fig. 4. Example of the execution of the optimisation algorithm. $i$ is the iteration counter. The total number of iterations is 68 .

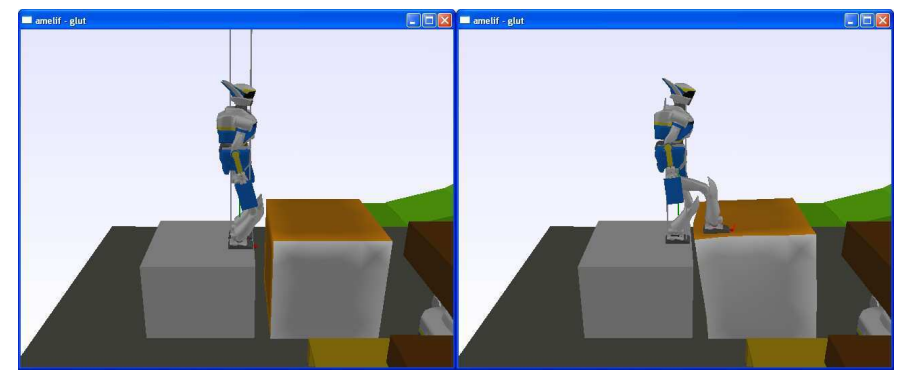

(a) Non-deformed configuration

(b) Deformed cube after taking a step

Fig. 5. HRP-2 taking a step on the deformable cube.

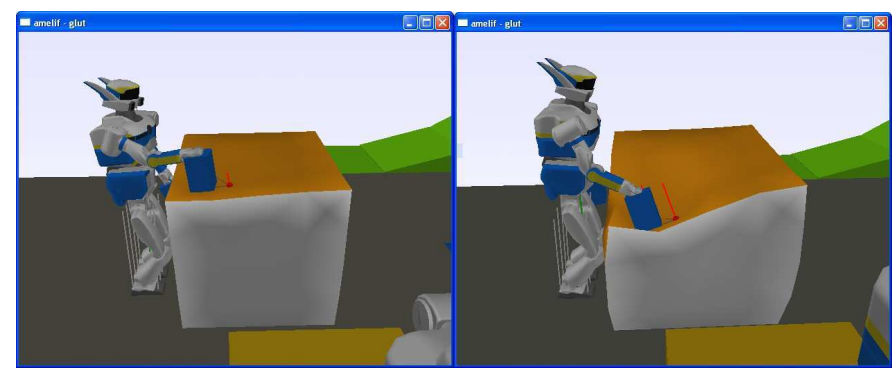

(a) $E=10^{6} \mathrm{~Pa}$

(b) $E=5 \times 10^{5} \mathrm{~Pa}$

Fig. 6. Resulting configuration with different Young's mudulus $E$.

\section{CONCLusion ANd Future Work}

We extended our multi-contact static posture planning optimisation framework to take into account non-rigid linearelasticity-based deformable model as a possible contact support. The linear behaviour made it possible to derive the gradient of the nodal reaction forces with respect to the configuration of the robot, which defines the boundary conditions of the deformation.

One limitation of this approach resides in its non applicability to the planning of the whole sequence of postures in the framework of contact-before-motion planning in its continuous formulation as presented in [2]. The reason is that the position of the contact print $(x, y, \theta)$ on the deformable surface should be specified and fixed beforehand in the current approach. If we were to keep this position $(x, y, \theta)$ as an optimisation variable, then the reaction forces would not any more be continuous functions of the configuration since the set $\mathscr{E}$ of the finite elements belonging the nonfixed contact print $S_{\text {print }}$ would vary in a discrete noncontinuous way. Thus it is not possible to use a finiteelement-based approach to plan for the sequence of postures under continuous search of the best positions of the contacts.

One way to overcome this limitation is to resort to a contact-before-motion planning approach in its prediscretized contact positions formulation as in [4], where we pre-process the environment by sampling a finite set of possible contact positions (fixed) on the environment, in particular on the deformable support, and perform a discrete search along these sampled positions. The approach presented in this paper is thus suitable in this case.

Finally, one remaining difficulty lies in the formulation of the collision-avoidance constraint with deformable objects.

\section{ACKNOWLEDGEMENT}

This work is partially supported by Japan Society for the Promotion of Science (JSPS) Grant-in-Aid for Scientific Research (B), 22300071, 2010.

\section{REFERENCES}

[1] K. Bouyarmane and A. Kheddar, "Static multi-contact inverse problem for multiple humanoid robots and manipulated objects," in Proceedings of the IEEE-RAS Int. Conf. on Humanoid Robots, 2010.

[2] - "Multi-contact planning for multiple agents," in Proceedings of the IEEE-RAS Int. Conf. on Robotics and Automation, 2011.

[3] A. Escande, A. Kheddar, and S. Miossec, "Planning support contactpoints for humanoid robots and experiments on HRP-2," in Proceedings of the IEEE/RSJ Int. Conf. on Intelligent Robots and Systems, 2006.

[4] K. Hauser, T. Bretl, and J.-C. Latombe, "Non-gaited humanoid locomotion planning," in Proceedings of the IEEE-RAS Int. Conf. on Humanoid Robots, 2005.

[5] M. Bonnet and A. Frangi, Analyse des solides deformables par la methode des elements finis. Ecole Polytechnique, 2005.

[6] J. R. Magnus and H. Neudecker, Matrix Differential Calculus with Applications in Statistics and Econometrics. Wiley, 1999.

[7] C. T. Lawrence and A. L. Tits, "Nonlinear equality constraints in feasible sequential quadratic programming," Optimization Methods and Software, vol. 6, pp. 265-282, 1996.

[8] A. Wachter and L. T. Biegler, "On the implementation of an interiorpoint filter line-search algorithm for large-scale nonlinear programming," Mathematical Programming, vol. 106, pp. 25-57, 2006.

[9] K. Kaneko, F. Kanehiro, S. Kajita, H. Hirukawa, T. Kawasaki, M. Hirata, K. Akachi, and T. Isozumi, "Humanoid robot HRP-2," in Proceedings of the IEEE Int. Conf. on Robotics and Automation, 2004

[10] J. Barbic and D. L. James, "Real-time subspace integration for st.venant-kirchhoff deformable models," ACM Transactions on Graphics, vol. 24, no. 3, 2005.

[11] Y.Zhuang and J. Canny, "Real-time global deformations," in Proceedings of the Workshop on Algorithmic Foundations of Robotics, 2000. 